\title{
Traffic Characterisation using Optical Based Packet Switches with Poisson and Fractal Traffic Sources
}

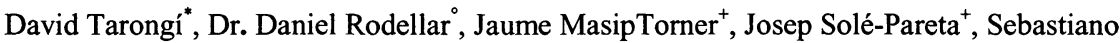 \\ Borgione $^{+}$ \\ *TCOM, EPFL, Telecommunications Laboratory, Ecole Polytechnique Federale de Lausanne, Switzerland. \\ 'SWISSCOM AG, Corporate Technology, Switzerland \\ + CCABA, UPC, Integrated Broadband Communications group, Universitat Politècnica de Catalunya
}

\section{INTRODUCTION}

Local area networks traffic and Internet traffic have been analysed for the last 25 years to obtain accurate characteristics that will be used in traffic models. Traditionally, traffic sources were simulated using a Poisson process model, that had the advantage of being easily generated. But as real traffic has been studied, it has been shown that real traffic properties differ from Poisson traffic properties. When designing new communication systems, different alternatives have to be compared with respect to performance, as a first step. In particular when no implementations is available, modelling is often the only way to evaluate quantitatively the alternatives. Besides a model for the system operation, there is a need for traffic models describing the characteristics of the load of the system resource.

Current Internet backbones require high-capacity transport networks as well as highcapacity packet routers in order to properly handle large amounts of traffic fluxes and the optical technology is taking a significant role to achieve this objective. Fiber transport capacities have reached the terabit per second range thanks to wavelength multiplexing techniques. To avoid capacity gaps with the Wavelength Division Multiplexing (WDM) transport network, packet routers need throughput capacities of the same order of magnitude ${ }^{[1]}$. On one hand, the introduction of optical functions provides the possibility of reaching high throughputs avoiding bottlenecks coming from the high electronic router complexities. On the other hand, all optical routers match the packet routing and WDM network capabilities in a single layer. Optical buffers have special characteristics with respect to conventional electronic buffers, and thus need a special analysis ${ }^{[2]}$, as they take profit of the advances in optical technology to implement all optical routing and buffering functions. The complexity of the optical buffer is independent of the total buffer capacity and its performance highly depends on the internal delay line configuration.

In this paper we aim to characterise the traffic that goes through an optical buffer to find out a delay line configuration that minimises the buffer complexity while maintaining the overall router performance. An optical buffer has been simulated with Poisson and self-similar traffic sources types. The traffic generators are completely characterised in terms of their packet inter-arrivals probability and their packet size distribution. These sources fill the optical buffer queues and the output traffic is then analysed.

The original version of this chapter was revised: The copyright line was incorrect. This has been corrected. The Erratum to this chapter is available at DOI: 10.1007/978-0-387-35491-0_28 


\section{OPTICAL PACKETS BUFFERING}

All optical routers will need optical buffers to solve packet contention. An all optical router with $N$ input/output fiber ports is considered and the set of $D$ transmission delay lines inside the optical router is called the optical buffer. A possible buffer configuration directly connects each subset of $D / N$ delay lines to one output. The resulting all-optical router configuration is output buffered.

Figure 1 shows an output optical buffer made up with 5 fiber delay lines. The number of optical delay lines in the output buffer determines the number of incoming packets can be loaded simultaneously towards a required output. The length of the longest delay line determines the total output buffer capacity. Note that the number of parallel accesses determines the complexity of the optical buffer and it is independent of the optical buffer capacity, which is the longest delay line. No optical packet synchronisers are considered and all fibers only contain a single wavelength for shake of simplicity (no WDM).

Each fiber delay line in the subset can have an arbitrary length. Each delay line introduces a different time shift on the scheduled output time. Packets that contend simultaneously for the same output avoid collision using a different time shift path. Incoming packets are always routed to the shortest available delay lines. One delay line can accept an incoming packet when:

- There is no other packet that is being loaded in the same delay line, and,

- Packets loaded on other delay lines are not scheduled with the same output time.

The time shift model in Figure 1 summarises all the available time shifts where incoming packets can be scheduled, one for each delay-line. According to this model, optical buffers with the same complexity (number of delay lines) can have different access distributions, i.e. different time shifting patterns. Figure 2 shows three possible configurations of an optical buffer, one with fixed steps, one with double fixed steps, and one with increasing step lengths.

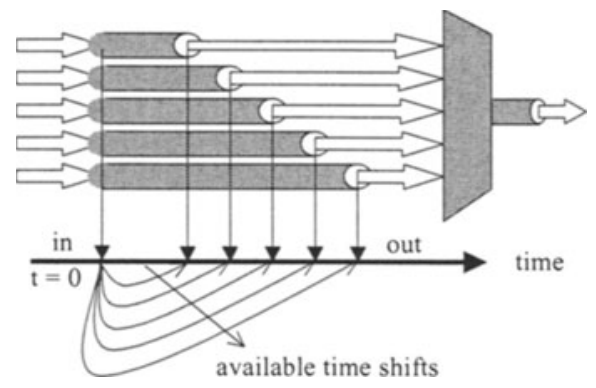

Figure 1: An optical buffer made up with 5 optical packets. The length of the longest delay line determines the total capacity of the optical buffer. 


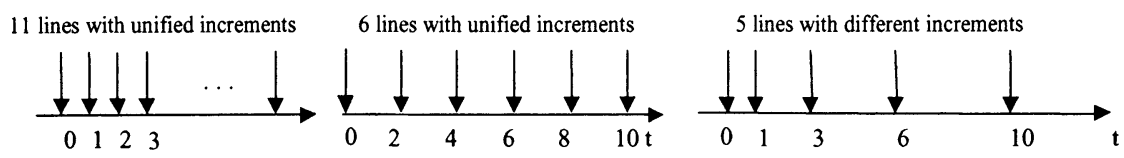

Figure 2: Possible configurations of an optical buffer

\section{TRAFFIC-DROP PRIORITY}

When the number of incoming packets increases to the congestion point, some of these packets will be lost. To determine which of them are going to be discarded, some traffic-drop priority settings have been defined. The traffic-drop priority scheme works as follows: if the network is not congested there is no difference in how the nodes treat each packet (e.g. no packet discard); and the packets are forwarded in the same order they are received. When congestion occurs best effort packets are discarded first, mitigating the congestion and leaving resources for the assured service packets.

An example of how this strategy can be implemented is given in Figure 3a. When a packet is received, the output port of the OPN node (and the associated queue) is determined. If the corresponding queue depth exceeds a given threshold N1 (congestion is imminent), best effort packets are discarded while assured packets are correctly sent into the queue.

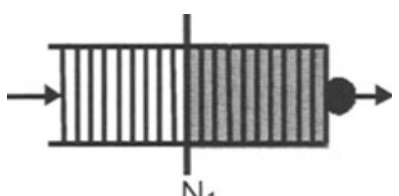

(a)

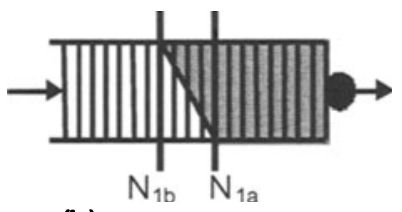

(b)

Figure 3: Output buffer with drop priority based on: (a) a threshold, (b) a RED policy.

This threshold mechanism can also be associated with a Random Early Discard (RED) strategy, providing a real advantage in the presence of optical packets carrying TCP/IP datagrams (Figure 3.b). When the buffer occupancy reaches the threshold N1a, the discard probability for a best effort packet begins to be more than zero and its value is increased to 1 when the buffer occupancy reaches the N1b value. In excess of this value only the assured quality packets can be buffered and all the best effort packets are discarded. It is worth noticing that in both cases (plain drop priority and RED) packets are discarded at the input of the OPN node: once packets have been entered the node, they undergo no scheduling modifications. This fits with the particular structure of optical buffers at OPN nodes, in which deleting a packet that has already entered the buffer is difficult and inadvisable. 


\section{TRAFFIC SOURCES}

Three different types of traffic sources have been implemented ${ }^{[3]}$ to feed the optical buffer. The first scheme is a synchronous and periodic source that is absolutely not realistic but can be very useful for the programmers to follow easily the packets inside the optical buffer, and to determine the accuracy of the implemented model. Another scheme implemented is a Poisson model for the packet arrivals, and even if it is not a realistic model for data network traffic, it has been used traditionally for traffic generation. A realistic model, based on the superposition of several Fractal Renewal Processes (FRP) is also implemented and characterised.

Each packet is generated by the sources at a given time, according to the generation distribution selected, and with a given packet size. The packet sizes can be either fixed or with a distribution taken from real Ethernet traces ${ }^{[4]}$.

\section{TRAFFIC CHARACTERISATION}

Several simulations are carried out with different traffic sources and the traffic is characterised at the input sources and the output of the optical buffer. Results of the simulation using each generation model for the packet inter-arrivals and a packet size distribution from real Ethernet traces are presented in the following paragraphs. The generated traffic is analysed. In the case of a Poisson process the mean and the variance of the traffic are related and correspond to the expectation defined by the number of packets per second generated. In the case of a self-similar (or fractal) process ${ }^{[5][6]}$, a variable $X$ is exactly self-similar with scaling parameter $H$ (Hurst parameter), if for all levels of aggregation $m$,

$$
X^{(m)}=m^{H-1} X
$$

Where $X^{(m)}$ is the average of $m$ consecutive values of the variable $X$. The resulting log-log representation of $\operatorname{Var}\left(X^{(m)}\right)$ versus $m$ is called variance-time plot. Intuitively, the critical characteristic of self-similar traffic is that there is no natural length of a "burst". At every time scale ranging from a few milliseconds to minutes and hours, similar-looking traffic bursts are evident. Aggregating streams of such traffic typically intensifies the self-similarity ("burstiness") instead of smoothing it. The words fractal and self-similar must be read in this text as synonymous.

To perform the simulations:

- The buffer was implemented with 5 delay lines, with delays going from $10 \mathrm{~ms}$ to $50 \mathrm{~ms}$ in steps of $10 \mathrm{~ms}$.

- The discrimination criterion followed in case of congestion is described in the Traffic Drop Priority section, with 1 class of service. The min-max size parameters $\left(\mathrm{N}_{\mathrm{la}}\right.$ and $\left.\mathrm{N}_{\mathrm{lb}}\right)$ were 4 5.

- The channel bitrate at the output of the buffer was $10 \mathrm{Mbit} / \mathrm{s}$, to be coherent with the packet size histogram data obtained from real Ethernet traces. 
- The observed packet size probability distribution function is represented in the Figure 4. From this distribution, the mean packet size is $2.975 \mathrm{Kbit}$.

- The simulation time was set to allow the generation and treatment of 1000000 packets.

- In a first approach, only Poisson traffic generated by the aggregation of 10 sources was considered. In a most realistic situation, the simulations were performed on self-similar traffic provided by 10 sources. In each one of the input sources, the fractal traffic is obtained by the superposition of several Fractal Renewal Processes (FRP).

For low load scenarios the input and output traffic characteristics were almost identical, since the packet reordering and losses were minimal. Therefore, only the results for high load cases, very close to the congestion point and even with some packet losses are presented.

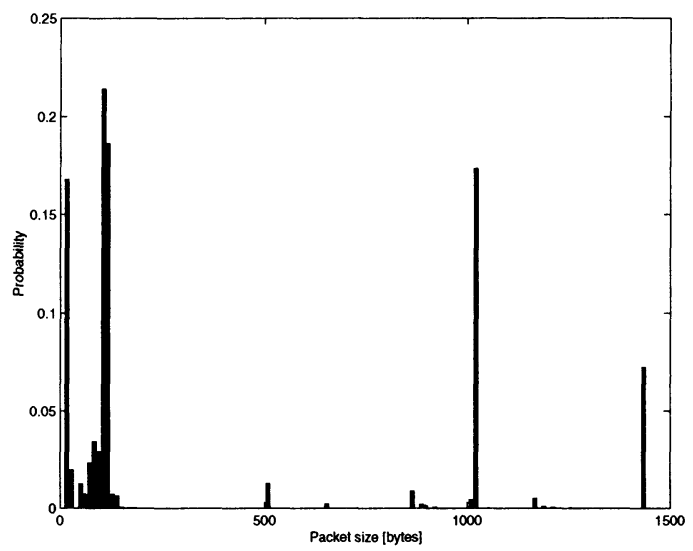

Figure 4: Probability of packet sizes, obtained from real Ethernet traces.

\subsection{Input Traffic Traces}

In this section the input traffic parameters for the simulations with Poisson and fractal traffic will be described. In both cases the number of sources was set to 10 , and the simulation time was about $407 \mathrm{~s}$ (1000000 packets in total). The simulated mean incoming bitrate was about 7.3 Mbit/s (7.291 Mbit/s in case of Poisson, and 7.308 Mbit/s for fractal case), which leads to an utilization factor of approx. $73 \%$ (input traffic: 0.73 Erlang). The interarrival time probability distribution for each one of the 10 sources is depicted in figures 5.a (Poisson traffic) and 5.b (fractal traffic). We can notice that, in case of Poisson traffic, the curve is well fitted by an exponential function. The mean value for the interarrival time, considering the aggregation of all the sources was $0.4 \mathrm{~ms}$ ( $4 \mathrm{~ms}$ for each source taken independently). 

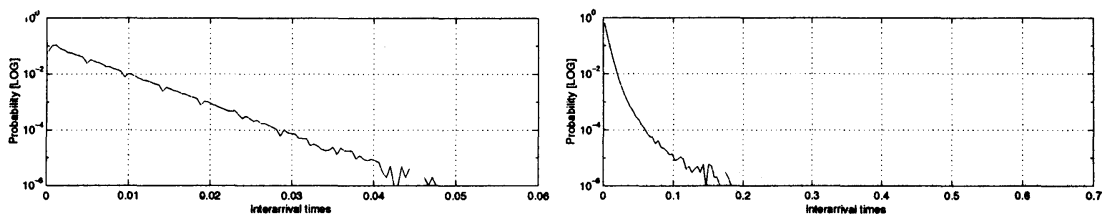

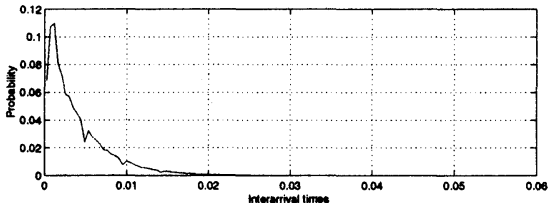

(a)

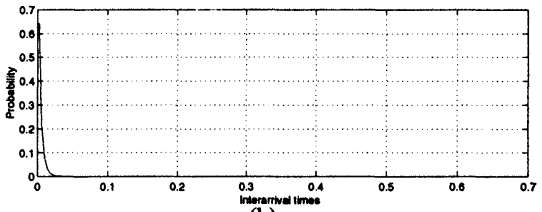

(b)

Figure 5: Interarrival time probability distribution, in logarithmic and linear scales, for each: (a) Poisson traffic source, (b) fractal traffic source.

To verify the self-similarity of the traffic provided by each fractal source, the Hurst parameter has been calculated, and the variance-time plot has been obtained from the input traces and represented in the Figure 6.

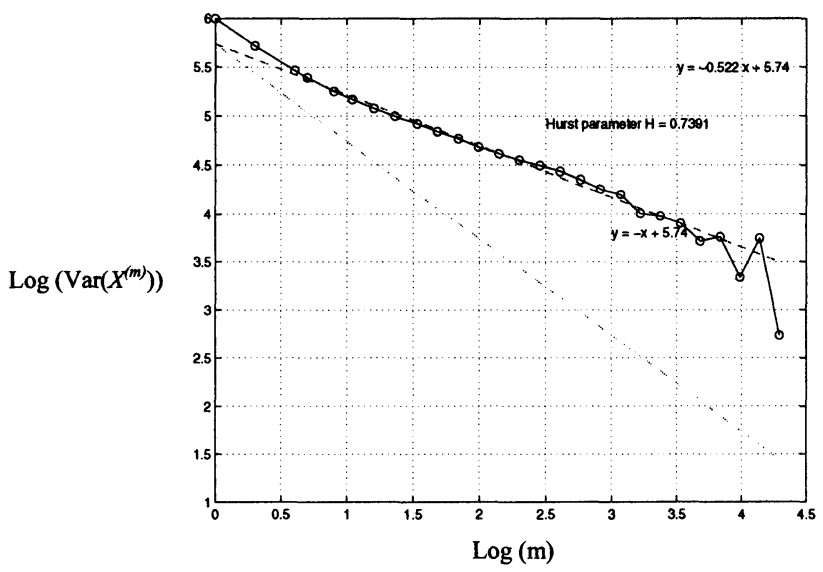

Figure 6: Variance-time plot of the traces. The lower dotted line corresponds to the variancetime plot expected for Poisson traffic.

In the Figure 7 a visualisation of the input traffic (packet generations for all nodes) for both traffic types during the first $10 \mathrm{~ms}$ of the simulation is provided. The packet generations are separated by sources on the vertical axis. We can appreciate the conflicts, which appear when some sources transmit their packets simultaneously. As stated in section 5.1, in both cases the traffic parameters were identical. The higher packet density observed in the Figure $7 \mathrm{~b}$ shows the "burstiness" of this traffic type, taking into account that the interval shown is very short compared to the whole simulation. 


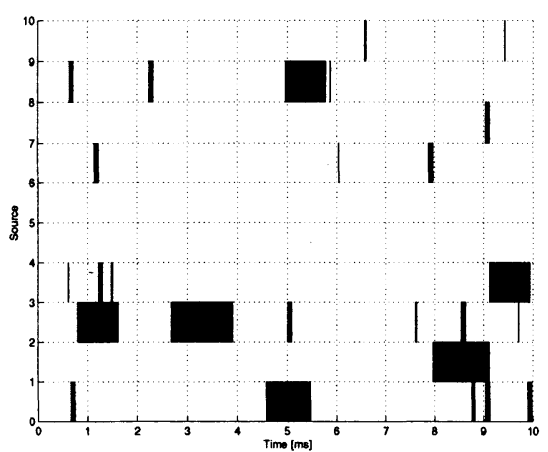

(a)

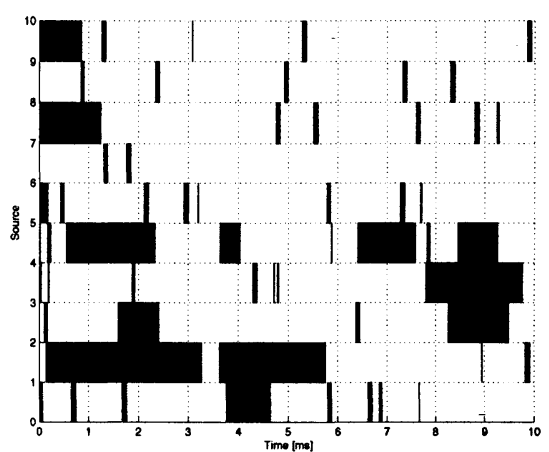

(b)

Figure 7: Packets visualisation by sources at the buffer input, considering: (a) Poisson traffic sources, (b) fractal traffic sources.

\subsection{Output Traffic Traces}

In this section, the results obtained from the simulations with the input traffic parameters specified before are presented. The Figure 8 provides the packets visualisation at the buffer output for the first $25 \mathrm{~ms}$, classified by sources like in Figure 7. As we can notice, there are no conflicts at the buffer output, since the packets have been properly delayed or lost. The most important parameters for the characterisation of the traffic output are presented in the Table 1.

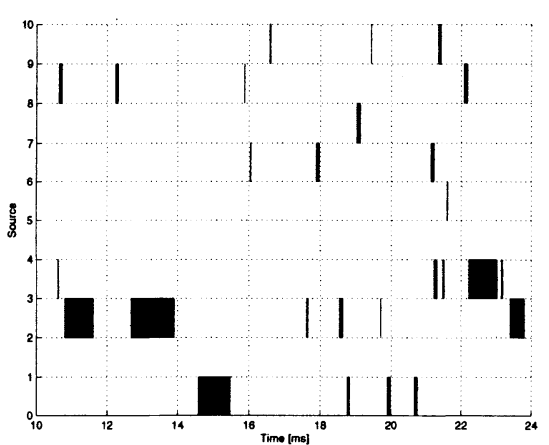

(a)

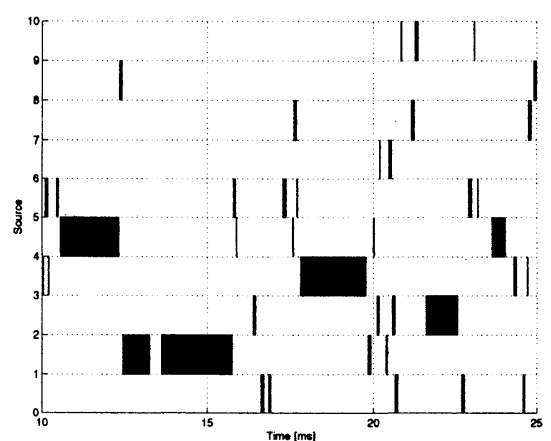

(b)

Figure 8: Packets visualisation at the buffer output, considering: (a) Poisson traffic sources, (b) fractal traffic sources. 


\begin{tabular}{c|c|c} 
Parameter & Poisson traffic & Self-similar traffic \\
\hline Output traffic (Erlang) & 0.6103 & 0.6095 \\
\hline Lost traffic (Erlang) & 0.1195 & 0.1213 \\
\hline Lost packets & $92329(9.2 \%)$ & $94964(9.5 \%)$ \\
\hline Mean packet size & 2742 & 2736
\end{tabular}

Table 1: Output traffic parameters, corresponding to the simulation with the input trace parameters provided in section 5.1

As we can see, the number of packets that have been discarded is slightly higher in the case of fractal traffic, due to the inherent burstiness of this more realistic traffic model. However, we have to notice that the difference is almost insignificant.

Figure 9 shows the interarrival time probability distribution at the buffer output. It is possible to observe the effects of the buffer on the traffic characteristics, more significant in the case of self-similar traffic. In this case, the distribution becomes even similar to a Poison one.
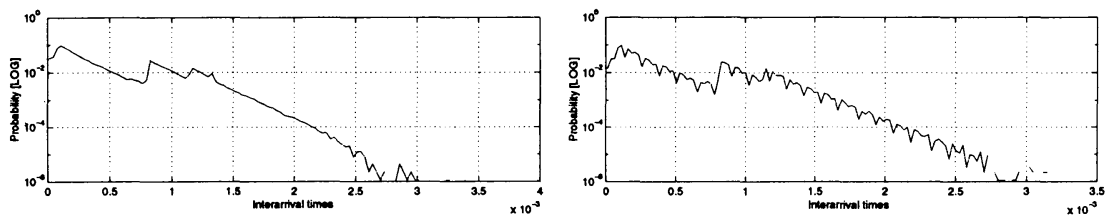

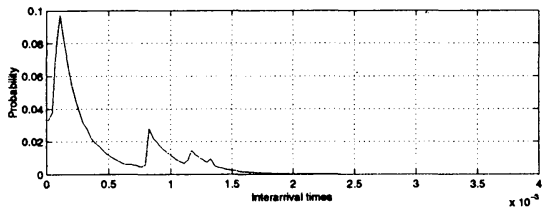

(a)

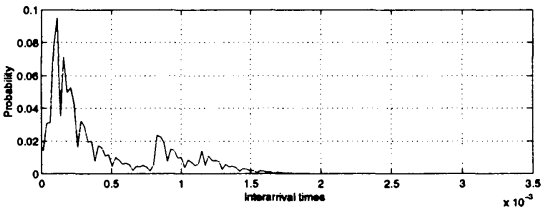

(b)

Figure 9: Interarrival time probability distribution, in logarithmic and linear scales, at the buffer output for: (a) Poisson input traffic, (b) fractal input traffic

The packet sizes distributions at the buffer output for both traffic models are represented in Figure 10. Comparing both graphics with the one at the input (Figure 4) it is possible to notice that the biggest packets have been more often discarded than the smallest ones. Therefore, the mean packet size at the output is around $2.740 \mathrm{Kbit}$ (2975 Kbit at the buffer input). Once more, the effect is very similar for both traffic models. 


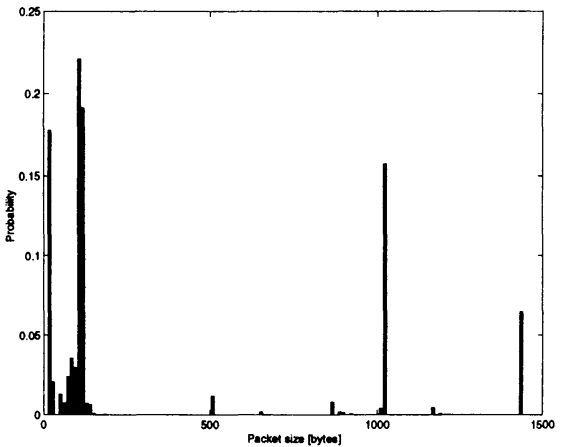

(a)

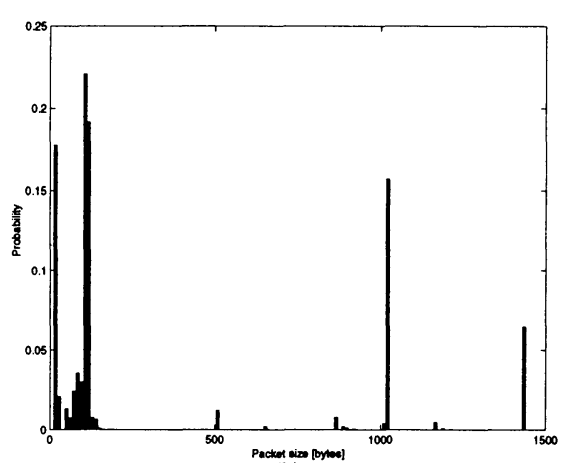

(b)

Figure 10: Packet size distribution at the buffer output, for the simulations with: (a) Poisson input traffic, (b) fractal input traffic.

\section{CONCLUSIONS \& FUTURE WORK}

A simple delay line configuration and more sophisticated configurations have been presented in this work. An optical buffer has been simulated with Poisson and fractal source types. The results for both cases have been shown.

The traffic generators are completely characterised in terms of their packet inter-arrivals probability and their packet size distribution. These sources fill the optical buffer queues and the output traffic has been analysed.

The impact of the optical buffer on the traffic characteristics is similar in both Poisson and self-similar input traffic cases. This shows that at high load scenarios the Poisson traffic model is suitable to be used in the characterisation of this kind of devices.

This study has provided a first approach in the characterisation of traffic using optical packets switching devices. Some improvements have to be taken into account in a future work:

- The buffer delay lines have to be designed to be the optimum and a statistical study has to be carried out to find out the optimal values for these delay lines (number of lines, delay distribution...). A first approach for this study could take into account the packet size distribution characteristics.

- A model of the optical for different Class of Services packets has to be created, and then exploited.

- The traffic generator will have to include a new parameter that will select the seeds to generate the packets, to allow several trace generations with different initialisation seeds.

- Different wavelengths must be considered, as well as the possibility of wavelength conversion, to be able to deal with WDM (Wavelength Division Multiplexing) devices. 


\section{ACKNOWLEDGMENTS}

This work has been supported and carried out in the framework of the COST Action Group 266 (Advanced Infrastructure for Photonic Networks) and the CICYT (Comisión Interministerial de Ciencia y Tecnologia) under contract TIC99-0572-C02-02.

Authors would like to acknowledge Prof. Pierre-Gérard Fontolliet and Dr. Cristian Bungarzeanu from the TCOM laboratory for their continued guidance and support. We would like also to thank the Swiss Federal Office of Education and Science for their financial support.

\section{REFERENCES}

[1] D.K. Hunter, I. Andonovic, M.C. Chia, “Optical Buffers for Multi-terabit IP Routers". 37 $7^{\text {th }}$ Annual Allerton Conference on Communication, Control and Computing. 22-24, September. 1999.

[2] Jaume Masip Torné, Josep Solé Pareta, Sebastiano Borgione, Bruno Bostica, Marco Burzio, "Providing Differentiated Service Categories in Optical Packet Networks", International Teletraffic Congress, 7-11 June 1999, IEE, 1999.

[3] D. Rodellar, "Performance Analysis of Multi-Channel Protocols for Optical Local Area Networks Exploiting Wavelength Division Multiplexing". Thesis of the Ecole Polytechnique Fédérale de Lausanne, 1999

[4] W. Leland, M. Taqqu, W. Willinger, and D. Wilson, "On the Self-Similar Nature of Ethernet Traffic (Extended Version)", IEEE/ACM Transactions on Networking, 2(1), February 1994, pp. 1-15.

[5] W. E. Leland and D. V. Wilson, "The measurement techniques used in making the traces are described in High time-resolution measurement and analysis of LAN traffic: Implications for LAN interconnection", Proc. IEEE INFOCOM'91, April 1991, pp. 1360-1366

[6] H. J. Fowler and W. E. Leland, "Local Area Network Traffic Characteristics, with Implications for Broadband Network Congestion Management", IEEE JSAC, 9(7), September 1991, pp.1139-1149 